\title{
PARA UNA SOCIOLOGIA \\ DEL RACISMO: \\ ANALISIS COMPARATIVO \\ DE LAS PAUTAS DE MATRIMONIO \\ ENTRE PERSONAS \\ DE RAZA BLANCA Y NEGRA
}

\author{
Ruth V. Aguilera
}

Universidad de Barcelona

RESUMEN. Existen maneras diferentes de analizar los prejuicios y las discriminaciones raciales. Uno de los mejores indicadores sociales es el matrimonio interracial (por ejemplo, entre razas negra y blanca), que sobrepasa los límites de los grupos establecidos mediante los procesos de racialización. El fenómeno del matrimonio interracial conlleva el definir y redefinir las nociones de raza, género y cultura en las vidas individuales, así como a nivel social y político. En el presente artículo se analizan algunos de esos conceptos e indicadores. Se estudian las tasas de matrimonios interraciales como un indicador significativo de la armonía en las relaciones raciales. A partir de esos datos empíricos se exponen las teorías existentes y posibles explicaciones del matrimonio interracial entre blancos y negros, utilizando los datos recientes de Estados Unidos y Gran Bretaña. Este artículo permite también analizar la dinámica de la racionalización en ambos países. Al final se añaden ideas provenientes de algunos de los principales estudios españoles.

«El problema del siglo xx es el problema de la línea de color -la relación entre las razas oscuras y las claras de las personas de Asia y Africa, en América y en las islas del mar.»

(DuBois, 1961: 23.)

El cruce de razas es un fenómeno antiguo. El Antiguo Testamento narra ya, por ejemplo, el matrimonio de Moisés y Zipporach. Sin embargo, es un tema que la Sociología «descubre» cada cierto tiempo, siempre con la ingenuidad de suponer que es un problema nuevo o distinto. El dilema 
resurge con el dilema de la película Jungle Fever, de Spike Lee: el noviazgo y el matrimonio interracial son frecuentemente utilizados como indicadores de la armonía de las relaciones raciales. Sin embargo, el fenómeno del noviazgo interracial es difícil de analizar debido a su falta de oficialidad, ya que normalmente no se registra.

El enfoque del presente artículo se centra en el fenómeno del matrimonio interracial. Los matrimonios interraciales son aquellos en los que dos personas de etnias o grupos raciales distintos se unen en matrimonio: en un enlace estable, legitimado y marital. El color de su piel es distinto y, por supuesto, «permanece» siempre diferente. Sean cuales sean sus religiones o sus nacionalidades, son personas de distintas razas (Gordon, 1964). En el presente trabajo, el término «matrimonio interracial» se refiere concretamente a las razas blanca y negra.

Al examinar la literatura científica sobre el matrimonio interracial entre personas blancas y negras se observa que se utilizan términos conceptuales diversos para designar este tipo de relaciones: «matrimonio mixto», «matrimonio interracial» y «matrimonio interétnico». La utilización de uno u otro término depende del punto de vista que el autor/a asuma en su obra. Por otro lado, la opinión pública considera que esas relaciones sociales son relaciones raciales.

La mayoría de la bibliografía internacional sobre este tema se publica en países anglosajones. El presente artículo es un análisis comparativo de las pautas recientes de matrimonio interracial en Gran Bretaña y Estados Unidos con algunos datos preliminares de España. Estados Unidos ha presenciado un proceso de segregación dentro de su propio territorio, y Gran Bretaña fuera de él. Sin embargo, estos dos países tienen algunas similitudes. En primer lugar, ambos son catalogados como sociedades capitalistas avanzadas fundamentadas sobre democracias liberales. En segundo lugar, la esclavitud y la explotación de mano de obra extranjera ha jugado un rol destacado en su desarrollo económico. En tercer lugar, existen privilegios de exclusividad «blanca», y la discriminación sistemática por el color y la raza es un factor social importante. Ambos países cuentan con una tasa considerable de población con actitudes etnocéntricas. Sin embargo, la diferencia principal entre estos dos países anglosajones es la dinámica de la «línea de color» (color line) que caracteriza la experiencia de la raza negra en cada país (Small, 1991: 8).

Históricamente, ambos países presentan un discurso elaborado sobre la negación de los matrimonios interraciales. En los Estados Unidos, los matrimonios entre personas blancas y negras estaban prohibidos por las leyes de antimestizaje. Todavía a principios de 1967 existían leyes de antimestizaje en dieciséis Estados norteamericanos. Se abolieron por la Corte Suprema en 1967 en el juicio Lovings vs. Commonwealth of Virginia. Prueba de ello son las manifestaciones del Ku Klux Klan en 1967 ante los cines que proyectaban la película de Stanley Kramer Adivina quién viene esta noche a 
cenar (Guess Who is Coming to Dinner), donde Khaterine Hepburn interroga angustiada a su hija sobre si había pasado la noche con su novio negro. Aunque en Gran Bretaña no existe una ley comparable a la de antimestizaje en Estados Unidos, sin embargo, el matrimonio interracial se consideró un tabú dentro de las ideologías racistas (Bagley, 1981). El miedo y los prejuicios hacia la «mezcla racial» alcanzan su punto de máxima intensidad en el siglo XIX, y actualmente aún persiste en varias instituciones y grupos sociales británicos.

Lo que parece claro en la literatura es el incremento de las tasas de matrimonios interraciales, tanto en Estados Unidos como en Gran Bretaña (Bagley, 1979: 1). Pero la hostilidad hacia los matrimonios interraciales es todavía considerable, y es clave para el análisis de las actitudes racistas. La medición del matrimonio con la variable de cruce de etnias o razas utiliza un índice de matrimonios interraciales que fue diseñado por primera vez por Savorgnan en 1950. Este índice mide el grado de matrimonios interraciales dentro de un ránking de $+1,00$ (todos los matrimonios dentro del mismo grupo racial) a $-1,00$ (todos los matrimonios fuera del grupo). Los datos utilizados en Gran Bretaña para medir este índice se extraen del censo de 1971. A pesar de que ese censo representa una mejora considerable respecto a los anteriores, especialmente en el área donde se registran los inmigrantes de la Nueva Commonwealth y sus descendientes, existen todavía inconvenientes con sus datos.

El problema principal es la información proporcionada indirectamente para determinar la raza de las personas por medio de su lugar de nacimiento. No tienen en cuenta los europeos nacidos, por ejemplo, en la India, ni tampoco los negros o asiáticos nacidos en Gran Bretaña. Los inmigrantes nacidos fuera de Gran Bretaña con padres británicos son bastante numerosos entre los indios, mucho menos numerosos entre los paquistaníes y relativamente poco comunes entre las personas procedentes de las Indias Occidentales. Las cifras pueden verse en la tabla 1 (Bagley, 1979).

Otros datos proporcionados por la Office of Population Censuses and Surveys (1979) ponen de relieve que alrededor de un 20 por 100 de las personas nacidas en la India y ahora residentes en Gran Bretaña son, de hecho, «étnicamente» europeas. Por esa razón, las tasas matrimoniales interraciales de los/as indios en Gran Bretaña son imposibles de calcular con precisión. Pero, según Bagley, aún es posible calcular tales índices para africanos, indios occidentales y paquistaníes (varones y mujeres en todos los casos), pues sólo representan un 5 por 100 o menos de las personas actualmente residentes en Gran Bretaña y «étnicamente» europeas. Sin embargo, es probablemente la última vez que va a ser posible dicho cálculo debido al crecimiento del número de personas de minorías étnicas nacidas en Gran Bretaña y ahora en matrimonios fecundos. Este problema estadístico fue especialmente grave en el censo de 1966 . El censo de 1971 reduce por primera vez tal incertidumbre -aunque no la elimi- 


\section{TABLA 1}

Proporción de matrimonios interraciales fecundos en Gran Bretaña que incluyen una persona paquistani, india-occidental o africana, con una europea (En porcentajes)

\begin{tabular}{|c|c|c|c|c|c|c|c|c|c|c|c|c|}
\hline \multirow[b]{3}{*}{ Persona } & \multicolumn{12}{|c|}{$\overline{A n ̃ o s}$} \\
\hline & \multicolumn{3}{|c|}{1969} & \multicolumn{3}{|c|}{1971} & \multicolumn{3}{|c|}{1975} & \multicolumn{3}{|c|}{1976} \\
\hline & Varón & Mujer & Total & Varón & Mujer & Total & Varón & Mujer & - Total & Varón & Mujer & Total \\
\hline India occidental & 13,7 & 3,5 & 17,3 & 16,5 & 4,7 & 21,2 & 21,2 & 7,8 & 29,5 & 24,2 & 8,7 & 33,0 \\
\hline Africana ............... & 10,5 & 6,2 & 16,8 & 12,7 & 7,5 & 20,2 & 12,2 & 8,8 & 21,1 & 10,8 & 8,5 & 19,3 \\
\hline Paquistaní .......... & $\ldots$ & $\ldots$ & $\ldots$ & $\ldots$ & $\ldots$ & $\ldots$ & 5,3 & 1,4 & 6,8 & 5,0 & 1,4 & 6,3 \\
\hline
\end{tabular}

Funve: Office of Population Censuses and Surveys, Londres.

na- mediante la consideración no sólo del lugar de nacimiento, sino también del país donde nacieron sus padres (padre y madre).

A partir del análisis del censo británico de 1971, Richard T. Schaefer (1981) diseña una tabla de los índices de matrimonios interraciales en Gran Bretaña. La tabla 2 deduce que existe poca variabilidad entre los distintos grupos étnicos. Siguiendo la tabulación de Savorgnan, $+1,00$ equivale a que todos los matrimonios se consumasen dentro del mismo grupo racial, y el cero significa la casi nulidad de mezcla de razas o etnias en el fenómeno interracial.

Se observa que el grado de endogamia es elevado. Los/as paquistaníes (la mayoría islámicos) tienen el índice más bajo de matrimonios interraciales. Los/as inmigrantes de la India (en su mayoría hindúes y algunos sikhs) tienen un índice comparativamente superior. Con los/as británicos de color nacidos en Gran Bretaña, el grado de interacciones interétnicas parece ser superior.

Las cifras de la tabla 2 apoyan las tesis de Bagley, quien utiliza los datos de nacimiento (de 1969) para calcular los porcentajes de matrimonios interraciales. Bagley demuestra que la endogamia es mayor entre los/as asiáticos, seguido de los/as indios occidentales, y menor entre los/as africanos, donde sólo el 57 por 100 de todos los matrimonios son endogámicos. Existe una proporción considerable de matrimonios interraciales en Gran Bretaña - una conclusión correcta si se limita a los modelos de matrimonio de padres con hijos/as nacidos recientemente en Gran Bretaña-. A pesar de que la tendencia se encamina hacia aumentar el grado de exogamia, el modelo británico debe aún describirse como endogámico respecto al matrimonio interracial.

Una característica a añadir a la realidad británica es la desconfianza 
TABLA 2

Matrimonio interracial para varios grupos en Gran Bretaña, 1971

\begin{tabular}{|c|c|}
\hline Grupo & $\begin{array}{c}\text { Tasa de matrimonio } \\
\text { interracial }\end{array}$ \\
\hline $\begin{array}{l}\text { Nacidos de color en Gran Bretaña ........... } \\
\text { Nacidos en la Nueva Commonwealth }\end{array}$ & 0,661 \\
\hline 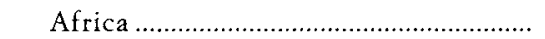 & 0,962 \\
\hline 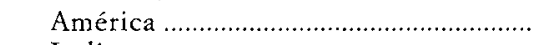 & 0,986 \\
\hline India & 0,956 \\
\hline 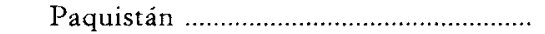 & 0,988 \\
\hline 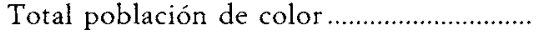 & 0,855 \\
\hline
\end{tabular}

Fuente: Censo británico 1971.

oficial del matrimonio interracial. Existen casos en archivo donde la policía ha entrado por la fuerza en hogares de parejas interraciales recién casadas. La razón para tales intrusiones es comprobar si la pareja está realmente viviendo junta. Incluso cuando ello es verdad, en ocasiones, el miembro de la pareja asiático o negro es arrestado o amenazado de deportación con el argumento de ser un inmigrante ilegal y de que su matrimonio es de «conveniencia».

Los datos empíricos de los Estados Unidos sobre matrimonios interraciales son más recientes y claros. Las estadísticas del Vital Statics of the United States son útiles para el tema que se está analizando. Se aprecia una cierta tendencia ascendente del número de matrimonios interraciales (véase tabla 3).

El Neewsweek (10 junio 1991) presenta la preocupación pública por este fenómeno. Según esa revista, en 1990 hay 211.000 matrimonios interraciales entre blancos y negros en los Estados Unidos, o, en otras palabras, son cuatro de cada mil parejas casadas; mientras que en 1970 sólo 1,5 de cada mil matrimonios es mixto. De los matrimonios interraciales en Estados Unidos hoy en día, el 71 por 100 son entre un varón negro y una mujer blanca, y sólo el 29 por 100 se refiere a varones blancos con mujeres negras. Ya es conocido que la versión varón-blanco con mujer-negra es poco usual, debido a la asociación estrecha entre clase social y raza.

Partiendo de las hipótesis de las teorías ilustradas por varios autores/as - contradictorias con los datos empíricos- sobre la permanencia intacta de la línea de color, ¿cómo se puede explicar tal incremento? ¿Está relacionado con las actitudes hacia la asimilación y el pluralismo? ¿Cómo afectan las políticas de antidiscriminación a ese incremento? ¿Es una relación de clases o de razas? Estos son algunos de los aspectos que conviene analizar con detenimiento. 


\section{TABLA 3}

Evolución de la proporción de matrimonios por raza en Estados Unidos (En porcentajes del total de matrimonios)

\begin{tabular}{|c|c|c|c|}
\hline \multirow[b]{2}{*}{ Matrimonios } & \multicolumn{3}{|c|}{$A \tilde{n} o$} \\
\hline & 1981 & 1984 & 1986 \\
\hline 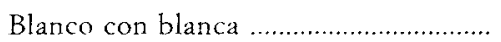 & 55,8 & 55,3 & 54,9 \\
\hline Negro con blanca & 0,34 & 0,37 & 0,45 \\
\hline Blanco con negra & 0,12 & 0,16 & 0,16 \\
\hline 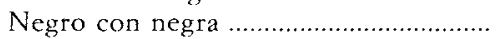 & 6,54 & 6,63 & 7,17 \\
\hline 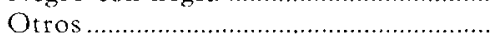 & 37.2 & 37,5 & 37,3 \\
\hline (1) & $\begin{array}{c}100 \\
(1.886 .396)\end{array}$ & $\begin{array}{c}100 \\
(1.904 .243)\end{array}$ & $\begin{array}{c}100 \\
(1.854 .744)\end{array}$ \\
\hline
\end{tabular}

Nota: Incluye todos los Estados excepto California, Colorado, Iowa, Massachussets, Michigan, Nueva York, Ohio y el DC.

Fuente: US Department of Health and Human Services, Vital Statistics of the United States 1981-86, (Washington, DC: GPO, 1989), vol. 3: «Marriage and Divorce», tabla 1.20 .

Existen varios factores a considerar en el análisis del modelo de matrimonio interracial en el contexto de las «relaciones raciales». El fenómeno del matrimonio interracial no puede ser explicado como un solo factor. Los condicionantes comunes a ambos países son la desaparición implícita de leyes discrimintorias, la acentuación de la estratificación de clases y la disminución de la disgregación. Existen dos modelos principales para el estudio de este fenómeno: el paradigma basado en las clases sociales y el de la etnicidad.

El paradigma basado en las clases sociales argumenta que «la división social que asume distintamente un carácter racial o étnico puede atribuirse o explicarse principalmente haciendo referencia a las estructuras y procesos económicos» (Hall, 1980). En la teoría basada en la clase social predominan tres enfoques: relaciones de mercado, estratificación social y conflicto de clases. Las definiciones más adecuadas del concepto de clase social a este planteamiento son las de Marx y Weber. La aproximación marxista de las relaciones de producción para ilustrar el fenómeno del matrimonio interracial es limitada por considerar exclusivamente la clase social en función de variables económicas. Sin embargo, puede utilizarse para explicar que la mayoría de trabajadores/as de raza negra tienen un empleo secundario, y a menudo reciben salarios inferiores que los/as trabajadores de raza blanca en lugares de trabajo similares.

Con referencia al matrimonio interracial, desde la definición weberiana de clase y su contribución a la teoría de la estratificación social (Clase, estatus 
y poder) parece más adecuada, pues teoriza el concepto de clase marxista, introduciendo a la vez el concepto del estatus. Estatus se refiere aquí a la educación, la renta, el prestigio ocupacional, las características económicosociales de los padres, etc. La educación es también un subfactor clave para designar la clase social. Esta aproximación trifactorial weberiana relaciona el estatus y el estilo de vida, siendo la renta un factor necesario pero no suficiente. S. M. Cohen establece dos consideraciones en cuanto a la relación entre nivel de educación elevado y asimilación racial: «Primero [...] las personas con mayor nivel educativo es más probable que se relacionen con personas de orígenes étnicos distintos cuando están en su último nivel educativo. Segundo, la educación liberal desarrolló individuos con valores y objetivos de mentalidad más abierta» (1974). Según Cohen, existe una tendencia positiva a medida que las personas adquieren más educación $y$ tienen mayor contacto interracial.

Otro componente significativo en el análisis del matrimonio interracial es la ocupación laboral. William J. Wilson, en su libro The Declining Significance of Race (1978), realiza una distinción entre la categoría de «clase» y la de «raza». Sugiere que la raza deja de ser el enlace entre las personas que lo consiguen y las menos afortunadas o «hermanos de clase desfavorecida» (Omi y Winant, 1990: 29). El orden en la jerarquía ocupacional permite la «movilidad social». El prestigio ocupacional es una de las variables determinantes del fenómeno del matrimonio interracial y sus características. Según los datos presentados por Wirth (1978), en general, los «varones de raza negra que se casan con mujeres de raza blanca suelen casarse con mujeres de raza blanca con un estatus ocupacional bajo» (Omi y Winant, 1990: 33). Al menos ése es el modelo en Filadelfia entre 1922 y 1944, según la investigación realizada.

El enfoque de la estructura social tiene una aplicabilidad directa en el análisis comparativo. En primer lugar, es importante tener en cuenta que la población de raza negra en Gran Bretaña representa un 4 por 100 de la población total, mientras que en Estados Unidos es el 12 por 100. En segundo lugar, las tasas de matrimonio interracial han aumentado más que proporcionalmente en Gran Bretaña que en Estados Unidos. Tercero, la dinámica política y económica de ambos países ha diferido considerablemente en las últimas décadas. La teoría de Blau de la estructura social propone los determinantes estructurales de las asociaciones sociales. Partiendo de la hipótesis de que la asociación social depende de las oportunidades de contactos sociales, Blau argumenta que cuanto mayores sean también las oportunidades de estos contactos, mayores serán las probabilidades de que se establezcan relaciones sociales justificadas (South y Messner, 1986: 1411). Blau considera cuatro elementos principales de la estructura social: tamaño del grupo, heterogeneidad, desigualdad y segregación.

Es importante explicar la diferencia en el incremento de las tasas de matrimonio interracial entre Estados Unidos y Gran Bretaña. La mayoría 
de negros/as en Estados Unidos son indígenas (es decir, nacidos en Estados Unidos). Existe una estratificación socio-económica dentro de la propia población de raza negra que debe tenerse presente; existe una clase media de raza negra con una representación política no despreciable. Ello es debido principalmente al Movimiento de Derechos Civiles (Civil Rights Movement) y de la propia trayectoria histórica contra el racismo. Por otro lado, Gran Bretaña no presenta una estratificación socio-económica significativa dentro de la población de raza negra, ni ésta posee un poder político representativo. Mientras que en los Estados Unidos la población de raza negra se considera indígena, en Gran Bretaña es considerada como inmigrante. Incluso la repatriación suele ser un aspecto frecuentemente discutido como solución a los problemas de las «relaciones raciales». En los Estados Unidos, la población de raza negra vive en comunidades distintas a las de la población inmigrante. Contrariamente, en Gran Bretaña todos los/as inmigrantes, incluyendo la población de raza negra, viven en las mismas áreas y van a las mismas escuelas bajo la categoría implícita de «inmigrantes». Así, la población negra en Gran Bretaña posee mayores oportunidades para un contacto racial con otras minorías de raza no-negra. Eso (según la teoría de Blau) facilita que se produzca más fácilmente el fenómeno del matrimonio interracial.

Una de las críticas principales a la teoría basada en las clases sociales es que no tiene en cuenta el concepto de «raza». Al evaluar el fenómeno del matrimonio interracial, la raza es definitivamente un factor importante, probablemente más relevante que el factor de origen nacional. El paradigma de etnicidad matiza en cierta medida las carencias del paradigma de las clases sociales. Se basa en dos principios: asimilacionismo y pluralismo cultural.

En el pasado, las minorías étnicas fueron analizadas en términos de su disolución como grupos de identidad y de su reducción a los valores de la cultura mayoritaria («blanca»). Esa perspectiva asume que la integración de minorías es un proceso natural en el cual se consigue igualdad y la discriminación, así como la segregación, se eliminan. El pluralismo cultural enfatiza la identidad de los grupos étnicos que constituyen los grupos minoritarios. Omi y Winant consideran que «este terreno incluye las dinámicas de incorporación de grupos minoritarios en la sociedad dominante. La teoría de la etnicidad, por lo tanto, se interesa principalmente por las cuestiones de identidad de grupo; con la resolución de tensiones entre las presiones gemelas de asimilación (disolución de la identidad del grupo) y pluralismo cultural (preservación de la identidad del grupo); y con las perspectivas para la interacción política vía los canales políticos normales» (Omi y Winant, 1990: 52). Desde un punto de vista asimilacionista, el incremento progresivo del matrimonio interracial en los Estados Unidos se considera como una consecuencia de la abolición de la legislación racista y la integración progresiva de las minorías de color en la sociedad blanca. 
La anulación de las leyes de antimestizaje se considera un paso para conseguir una sociedad no-racista. Además, intenta integrar a toda la población en unas condiciones de igualdad. Según los datos del censo norteamericano (Census Bureau Survey), «desde la desaparición de las últimas leyes de no-mestizaje hace casi veinticinco años, los matrimonios entre blancos y negros se han más que triplicado en esta nación, desde menos de 65.000 a 211.000» (Perry Lang, 1991). Desde el punto de vista de Myrdal, la abolición de las leyes de no-mestizaje es otro ejemplo del «credo americano» (democracia, igualdad y justicia), el cual integra a todos sus ciudadanos/as independientemente de su color. Asume que la disgregación y la no-discriminación sólo pueden conseguirse a través de una asimilación de los valores de la mayoría. Sin embargo, cuando se analiza la distribución regional de los matrimonios interraciales en Estados Unidos se observa que las discrepancias significativas no pueden explicarse a partir de las diferencias en el grado de asimilación. En cambio, se explican en relación al colonialismo y la resistencia de esos grupos hacia ese tipo de matrimonios.

Según el censo estadounidense de 1985, las tasas de matrimonios interraciales son superiores en el Oeste que en el Este y en el Sur (Center of Afro-American Studies). Las diferencias regionales, el tipo de matrimonios, los conflictos de identidad de las parejas y sus respectivas redes sociales y la movilidad de la pareja casada no se pueden explicar a partir de un punto de vista asimilacionista. Por el contrario, es necesario introducir el concepto de «pluralismo cultural», el cual subraya la identidad de los distintos grupos étnicos. El asimilacionismo es un discurso de derecho utilizado por la mayoría blanca, que permite la explicación del incremento de matrimonios interraciales acompañado de la abolición de las leyes de antimestizaje. No tiene en cuenta los valores culturales de los grupos minoritarios, su resistencia en la asimilación de los valores mayoritarios o la preservación de su identidad.

En Gran Bretaña, el incremento de los matrimonios interraciales es el resultado de la integración de los grupos minoritarios en el modo de vida del país. Según Small, en su artículo «Attaining racial parity in the United States and England: We got to go where the greener grass grows!» (1991), Gran Bretaña aparece más integrada física y socialmente que Estados Unidos. Por ejemplo, el 50 por 100 de la población negra actual ha emigrado de los países de la Nueva Commonwealth. Bagley (1981) señala que, gracias a que una gran mayoría de los inmigrantes son varones, les proporciona mayores oportunidades para establecer interacciones sociales con mujeres británicas blancas. Esta puede ser otra hipótesis para explicar por qué al principio más varones negros se casaban con mujeres blancas. Se observa que la tasa de matrimonios blanco/negro (ambas combinaciones: varón/mujer y mujer/varón) en Gran Bretaña es tres veces mayor que en Estados Unidos (Bagley, 1981). En Gran Bretaña, un 20 por 100 de la población negra y asiática están casados con una persona blanca. La Office 
of Population Census and London Surveys muestra que, en 1969, el porcentaje de matrimonios entre la población oriunda de las Indias Occidentales y los blancos/as era un 17,3 por 100 , y entre africanos/as y blancos/as un 16,8 por 100. En 1976, estas tasas aumentan hasta un 33,0 por 100 para los primeros y un 19,3 por 100 para los segundos.

Desde la aproximación asimilacionista no es posible explicar la política de inmigración hacia la población de color en beneficio del gobierno británico. Estos son discriminantes que fomentan el incremento del racismo y de la hostilidad en las relaciones raciales (Miles y Phizacklea, 1987). El incremento en los matrimonios interraciales no es una consecuencia de las leyes que desfavorecen las minorías de color, sino un fenómeno que debería observarse desde las prácticas sociales que se llevan a cabo en la interacción entre las minorías de color y la mayoría blanca.

Existen algunos problemas en las estadísticas británicas porque las personas están clasificadas desde el punto de vista de la etnia de nacimiento de sus padres. Los/as inmigrantes en Gran Bretaña junto con sus hijos/as son considerados del mismo grupo étnico. Por lo tanto, es difícil distinguir entre inmigrantes y niños/as nacidos en Gran Bretaña. La identidad de los inmigrantes y la de sus hijos/as difiere en el sentido de que los últimos se han socializado en Gran Bretaña. A la segunda generación se les permite (hasta cierto grado) asimilar algunas prácticas y valores culturales británicos.

En Gran Bretaña, un estudio realizado en los años setenta sobre las actitudes hacia el matrimonio interracial demuestra que sólo un 15 por 100 de las parejas se consideran a sí mismas culturalmente pluralistas y aceptan ese tipo de relación (Bagley, 1979). Con los datos de ese estudio se puede concluir que, a pesar del incremento que se ha producido en las tasas de matrimonio interracial, la hostilidad y la negación pública hacia el fenómeno de matrimonio interracial es latente. No sólo permanecen hostiles hacia los matrimonios, sino que también son reacios/as a aceptar a los hijos/as de matrimonios interraciales. Anne Wilson analiza los problemas de identidad de los hijos/as de matrimonios formados por dos razas distintas y llega a la conclusión de que «a pesar de la liberalización de las actitudes hacia el matrimonio interracial, los niños/as mezcla de dos razas se consideran aún como un grupo con desventajas psicológicas» (1987: 91). Debido a la falta de estudios más recientes sobre las actitudes de la población, no es posible saber con certeza si la tendencia del incremento del matrimonio interracial es consecuencia de una mayor aceptación y tolerancia de las minorías de color.

Las variables del paradigma de la etnicidad, tales como el asimilacionismo o el pluralismo cultural, responden a dos posiciones -etnocentrismo y relativismo cultural - que explican el incremento de matrimonios interraciales en su función de asimilación. Sin embargo, este paradigma no puede explicar elementos del paradigma anterior como la movilidad social o la posición dentro de una estructura socioeconómica. 
El matrimonio interracial es un suceso social, y el color de la piel puede influir sustancialmente en los prejuicios sociales y en el control social. Por lo tanto, las tasas de matrimonio interracial son un buen indicador de la armonía en las relaciones raciales. A pesar de que las tasas de matrimonio interracial se han incrementado en algunos países avanzados (como Estados Unidos o Gran Bretaña), todavía existe un largo camino por recorrer por lo que se refiere a mejorar relaciones raciales y procesos de tolerancia en la sociedad actual.

La transformación de los países de la costa norte de la ribera mediterránea de países de emigración en países de inmigración constata el éxito del largo proceso de reconstrucción y desarrollo económico de estos países. Países como Italia, España, Portugal y Grecia ejercen una fuerte atracción de emigrantes procedentes de Marruecos, Túnez y Egipto a través del Mediterráneo, e incluso a inmigrantes provenientes de países más distantes como Pakistán e Irán.

La dinámica del fenómeno migratorio español se puede diferenciar en tres etapas. A partir de los años sesenta se presencia un aumento regular de la inmigración que se sostiene hasta los años setenta (se pasa de los 60.000 inmigrantes a los 120.000). Durante los años setenta se produce un estancamiento del saldo migratorio. Y en los años ochenta se reanuda el proceso de inmigración. Según Cagiano (1990), los datos oficiales de 1987 contabilizan alrededor de 350.000 inmigrantes legales. Otros estudios sobre extranjeros/as en situación no legal estiman que éstos alcanzan una cifra similar.

El número de extranjeros establecidos en España está constituido por una parte de europeos que, en su mayor parte, pertenecen a colectivos de jubilados que reciben el dinero que les envían sus gobiernos, y suponen una fuente de ingresos para el Estado que los acoge. Por otro lado, figuran los inmigrantes activos procedentes de América Latina, Asia y Africa (en particular Marruecos). La tabla 4 describe la evolución de la población extranjera residente en España. Se aprecia un aumento notable de inmigrantes africanos. Cáritas cifra en 294.000 los residentes en España ilegalmente y procedentes de países pobres. Entre los africanos/as, sólo uno de cada cinco habla español, con las dificultades que ello significa a la hora de acceder a un trabajo e integrarse a la realidad española (El País, 10 de noviembre de 1992). Estos constituyen un grupo de mano de obra poco cualificada, de los cuales un 40 por 100 trabaja en la agricultura y construcción (J. M. Herrera, responsable de inmigración de Cáritas Española). Sin embargo, las corrientes de americanos y europeos ocupan a menudo puestos laborales de cualificación elevada.

C. Solé y E. Herrera (1991) realizan un estudio acerca de los trabajadores extranjeros en Cataluña (48 municipios) con un universo de 4.130 personas inmigrantes (228 cuestionarios codificados) procedentes de Gambia, Guinea-Bissau, Nigeria, Senegal, Marruecos, Filipinas y Pakistán. En ese 


\section{TABLA 4}

Evolución de la población extranjera residente en España (En cifras absolutas y porcentajes)

\begin{tabular}{|c|c|c|c|c|}
\hline \multirow[b]{3}{*}{ Pais de origen } & \multicolumn{4}{|c|}{$A \tilde{n} o s$} \\
\hline & \multicolumn{2}{|l|}{1980} & \multicolumn{2}{|l|}{1987} \\
\hline & Número absoluto & $\%$ & Número absoluto & $\%$ \\
\hline Reino Unido . & 23.773 & 11,88 & 55.981 & 16,52 \\
\hline Francia & 16.743 & 8,37 & 23.667 & 6,99 \\
\hline 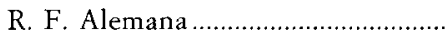 & 21.878 & 10,94 & 39.596 & 11,88 \\
\hline Portugal ................ & 24.367 & 12,18 & 31.048 & 9,16 \\
\hline Italia & 9.671 & 4,83 & 13.070 & 3,86 \\
\hline Otros & 28.670 & 14,33 & 53.535 & 15,79 \\
\hline 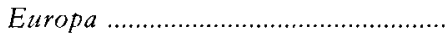 & 125.102 & 62,54 & 216.907 & 63,99 \\
\hline México & 2.361 & 1,18 & 3.115 & 0,92 \\
\hline Cuba …1...... & 7.720 & 3,86 & 5.785 & 1,71 \\
\hline Argentina & 8.506 & 4,25 & 14.130 & 4,17 \\
\hline Venezuela & 8.318 & 4,16 & 8.157 & 2,41 \\
\hline Chile & 4.052 & 2,03 & 6.264 & 1,85 \\
\hline 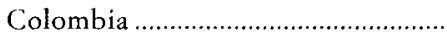 & 3.038 & 1,52 & 4.114 & 1,21 \\
\hline Otros & 9.397 & 4,70 & 14.689 & 4,33 \\
\hline 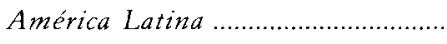 & 43.392 & 21,69 & 56.254 & 16,60 \\
\hline 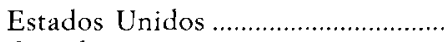 & 10.689 & 5,34 & 16.016 & 4,73 \\
\hline Canadá & 1.020 & 0,51 & 1.492 & 0,44 \\
\hline 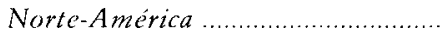 & 11.709 & 5,85 & 17.508 & 5,17 \\
\hline Marruecos & 3.095 & 1,55 & 11.312 & 3,34 \\
\hline 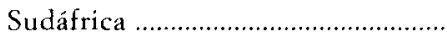 & 1.125 & 0,56 & 6.270 & 1,85 \\
\hline Otros & 680 & 0,34 & 1.364 & 0,40 \\
\hline Africa & 4.900 & 2,45 & 18.946 & 5,59 \\
\hline 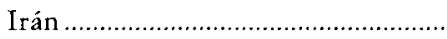 & 573 & 0,29 & 2.623 & 0,77 \\
\hline Resto países oriente & 4.389 & 2,19 & 4.146 & 1,22 \\
\hline 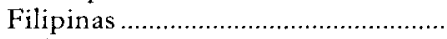 & 2.865 & 1,43 & 8.311 & 2,45 \\
\hline India & 2.430 & 1,21 & 4.933 & 1,46 \\
\hline (다. & 729 & 0,36 & 3.035 & 0,90 \\
\hline Otros & 2.254 & 1,13 & 4.078 & 1,20 \\
\hline Asia & 13.240 & 6,62 & 27.126 & 8,00 \\
\hline 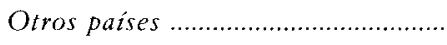 & 1.706 & 0,85 & 2.217 & 0,65 \\
\hline TOTAL & 200.049 & 100 & 338.958 & 100 \\
\hline
\end{tabular}

Fuente: F. M. Perez y A. I. Escribano, Population, 2, 1989. 
estudio incluye la variable matrimonio, lo cual nos permite analizar la situación para un área específica de España. No debe olvidarse que Cataluña ha sido históricamente un centro de atracción y confluencia de gente de diversa procedencia geográfica, que tantas veces ha interrumpido el viaje hacia otros países europeos. La tabla 5 constata el comportamiento de los matrimonios interraciales $\mathrm{y}$, consecuentemente, el nivel de integración de esta población. Se llega a la conclusión que sólo un 33 por 100 de la población extranjera que trabaja en Cataluña está casada. Cabe señalar que en su gran mayoría son varones, y representando el grupo de personas entre los 31 y 40 años el 71 por 100 de la población extranjera que trabaja en Cataluña (Solé y Herrera, 1991). A su vez, de éstos los casados el 84 por 100 lo hizo en su país e inmigró sin su familia. No existen, según estos datos, inmigrantes extranjeros/as procedentes de los países anteriormente enumerados casados con ciudadanos españoles o con ciudadanos de otros países. Eso es significativo del bajo nivel de integración de esta población en Cataluña, extrapolable al resto de España.

El racismo, la integración étnica y el pluriculturalismo son aspectos que están reapareciendo y realzándose en nuestra sociedad con el nuevo orden económico mundial y los nacionalismos emergentes. Los estudios desde la perspectiva sociológica son cada vez más numerosos, lo cual es indicativo de la trascendencia social de tales eventos.

\section{TABLA 5}

Situación de los trabajadores extranjeros residentes en Cataluña que están casados

(En porcentajes)

\begin{tabular}{|c|c|c|}
\hline & Frecuencia & $\%$ \\
\hline 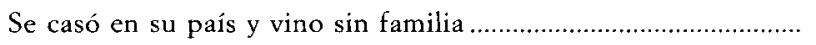 & 65 & 84 \\
\hline 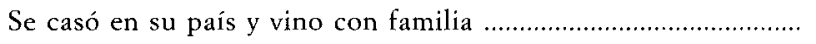 & 7 & 9 \\
\hline 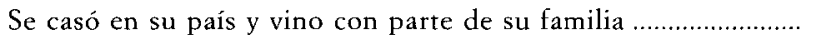 & 3 & 5 \\
\hline 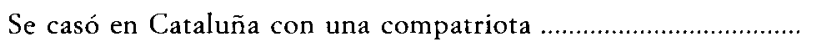 & 1 & 1 \\
\hline
\end{tabular}

Nota: Extranjeros se refiere a los provenientes de Gambia, Guinea Bissau, Nigeria, Senegal, Marruecos, Filipinas o Paquistán

Fuente: C. Solé y E. Herrera, Trabajadores extranjeros en Cataluña, Madrid: CIS, 1991. 


\section{BIBLIOGRAFIA}

BACiLEY, C. (1972): «Patterns of interethnic marriages in Great Britain», New Community, vol. 1 (verano), pp. 318-326.

- (1979): «Inter-ethnic marriage in Britain and the United States from 1970-77: A selected abstract bibliography», Sage Race Relations Abstracts, vol. 4, núm. 1, pp. 1-22.

- (1981): «Mixed marriages and race relations today», Patterns of Prejudice, vol. 15, núm. 1 .

Banton, Michael P. (1977): The Idea of Race, Londres: Tavistock.

- (1983): Racial and Etbnic Competition, Cambridge: Cambridge University Press.

Blau, Peter (1977): Inequality and Heterogeneity, Nueva York: Free Press.

Blau, Peter, y Duncan, O. D. (1967): The American Occupational Structure, Nueva York: Wiley.

BRown, Colin (1984): Black and White Britain: The Third PSI Survev, Londres: Heinemann.

CAGIANO DE AZLVEDO, Raimondo (1990): Inmigración, ayuda al desarrollo política inmigratoria europea: Los casos de Italia, España y Portugal, Barcelona: Itinera.

Calvo, Tomás (1990): ¿España racista?, Barcelona: Anthropos.

Center of Afro-American Studies (1990): «New trends in black American interracial marriage: The social structural context», Journal of Marriage and the Family, pp. 211219.

CoHEN, Steven M. (1974): Patterns of Interethnic Marriage and Friendship in the United States, tesis doctoral, Columbia University.

-- (1977): «Socioeconomic determinants on interethnic marriage and friendship», Social Forces, vol. 54, núm. 4 (junio).

Combate el racismo: Dossier de prensa, documentos y materiales de trabajo sobre racismo y xenofobia, Madrid: Instituto de la Juventud, Ministerio de Asuntos Sociales, 1992.

DuBors, William E. B. [1903] (1961): The Souls of Black Folk, Nueva York: Fawcett.

EATon, G., y MiLTon, J. (1985): Racial and Cultural Minorities, Nueva York: Plenum Press.

GoLDEN, Joseph: Characteristics of Negro-White Intermarried in Pbiladelphia, Florida State University.

Gordon, Albert I. (1964): Intermarriage, Boston: Beacon Press.

HAll, Stuart (1980): «Race, articulation, and societies structured in dominance», Sociological Theories: Race and Colonialism, París: UNESCO, $306 \mathrm{pp}$.

Hechter, Michael: «Group formation and cultural division of labour», American Journal of Sociology, vol. 844, núm. 22.

Informe Ford sobre el racismo en Europa, Madrid: Instituto Nacional de Servicios Sociales, Ministerio de Asuntos Sociales, 1991.

Jencks, Christopher (1992): Rethinking Social Policy: Race, Poverty and the Underclass, Cambridge: Harvard University Press.

LANG, Perry (1991): «Living in an interracial marriage», The New York.

Livi-Baccl, Massimo (1991): Inmigración y desarrollo: Comparación entre Europa y América, Barcelona: Itinera Cuadernos.

Miles, Robert, y Phizacklea, Annie (1987): White Man's Country, 2." ed., Londres: Pluto Press.

Myrdal, Gunnar [1944] (1962): An American Dilemma: The Negro Problem and Modern Democracy, Nueva York: Harper \& Row.

OMI, Michael, y WinANT, Howard (1990): Racial Formation in the United States, Nueva York: Routledge \& Kegan Paul.

PASEOE, Peggy: «Race, gender, and intercultural relations: The case of interracial marriage», Frontiers, vol. 12, núm. 1.

Rallu, J. L., y Blum, A.: European Population, a synthesis of European Population, vol. 1. SAvorgnan, Franco (1950): «Matrimonial selection and the amalgamation of heterogenous couples», Population Studies, vol. 3 (marzo), pp. 60-61.

Sirkofr, H. (1981): The Struggle for Black Equality (1945-80), Nueva York: Hill and Wang.

SchalFer, Richard T. (1981): «Intermarriage in Britain and the United States», Patterns of Prejudice, vol. 15, núm. 2. 
SMALL, Stephen (1991): «Attaining racial parity in the United States and England: We got to go where the green grass grows!», Saga Race Relations, vol. 16, núm. 2 (mayo).

- (1991): «Racialised relations in Liverpool: A contemporary anomaly», New Community, vol. 12, núm. 14 (julio).

SOLE, Carlota, y Herrera, Encarna (1991): Trahajadores extranjeros en Cataluña: ¿Integración o racismo?, Madrid: Centro de Investigaciones Sociológicas.

SoUth, J., Messner, Steven F. (1986): «Structural determinants of intergroup association: Interracial marriage and crime», American Journal of Sociology, vol. 91, núm. 6 (mayo), pp. $409-430$.

Vital Statics of the United States: Marriage and Divorce, 1981-86, Washington, DC: Department of Health and Human Services, vol. 3.

Wilson, Anne (1987): Mixed Race Children: A Study of Identity, Londres: Allen \& Unwin.

WILSON, William J. (1978): The Declining Significance of Race, 2." ed., Chicago: The University of Chicago Press.

Wricht, Erci O. (1978): «Race, class, and income inequality», American Journal of Sociology, vol. 33, núm. 6, pp. 1397. 